\title{
Aktuelle Studie belegt: Keine Hautreizungen durch Wäscheweichspüler
}

\section{Entgegen einer weit verbreiteten Meinung sind Weichspülmittel auch bei Atopikern verwendbar und verursachen keine Hautreizungen. Patienten mit einer Überempfindlichkeit gegenüber Textilien können von Weichspülern sogar profi- tieren: Bei Atopikern mit schlechtem Hautzustand führte die regelmäßige Behandlung der Wäsche mit diesen Pflegemitteln sogar zu einer Verbesserung des Hautbildes, so die Ergebnisse einer aktuellen Untersuchung.}

Verbreitet ist auch heute noch das Vorurteil speziell unter Allergikern bzw. Neurodermitikern, Weichspüler seien „pure Chemie“ und damit per se schädlich für die empfindliche Haut. Die Wäschepflegemittel standen daher immer wieder in dem Verdacht, Hautveränderungen und -irritationen auszulösen. Wissenschaftlich belegt wurde diese Behauptung allerdings nie - im Gegenteil, verschiedene Untersuchungen ließen eher auf einen protektiven Effekt auf den Hautzustand der Patienten schließen.

\section{Testpersonen: Atopiker mit Hautläsionen}

Die "Studie zur Wirkung von Weichspülern auf die Haut von Atopikern" am Beispiel von Lenor Care nahm sich dieser Problematik erneut an. Unter der Leitung von Th. Bieber (Wilhelms-Universität Bonn) in Zusammenarbeit mit der Deutschen Haut- und Allergiehilfe (DHA) wurden 50 Atopiker mit unterschiedlich stark ausgeprägten Hautläsionen in die Untersuchung eingeschlossen. Die Probanden wendeten Wäscheweichspülmittel (hier Lenor Care) regelmäßig an.

Ergebnis: 67\% der Probanden, die zu Beginn der Studie unter einem besonders schlechten Hautbild litten, be-
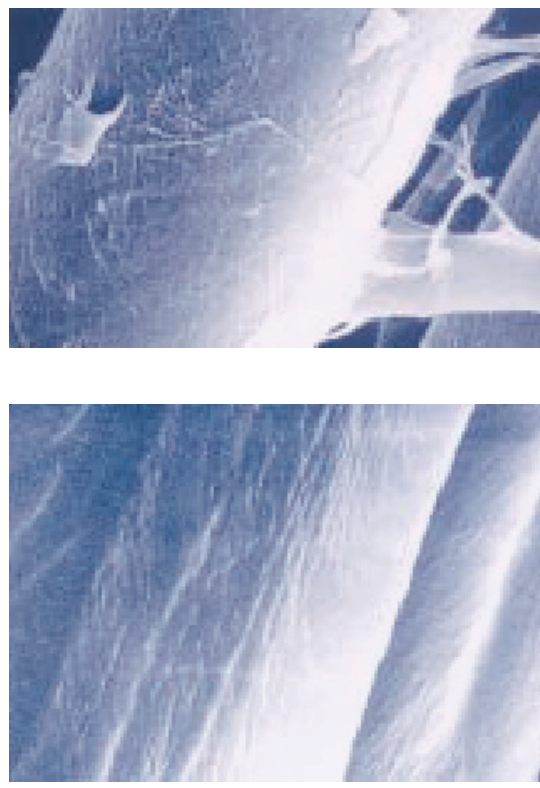

Effekt von Weichspülmitteln auf Textilfasern: Die Oberfläche von unbehandelten Fasern ist deutlich rauher (Abb. oben) als die mit Weichspülmitteln behandelte Faser (Abb. unten). Diese hat ein geringeres mechanisches Reibungspotential, was sich insbesondere bei empfindlicher oder geschädigter Haut vorteilhaft auswirkt. (Fotos: Lenor Care, Procter \& Gamble $\mathrm{GmbH}$ )

urteilten ihren Hautzustand während bzw. nach dem Test als besser, 25\% registrierten keine Veränderung unter dem
Weichspüler und $8 \%$ berichteten über eine Verschlechterung ihres Hautzustandes, was aber nach Ansicht der Prüfdermatologen nicht auf den Gebrauch des Weichspülmittels zurück zu führen war (Bieber Th, Fried J; Publikation in Vorbereitung).

\section{Weichgespülte Wäsche verbessert Hautzustand}

Ähnliche Ergebnisse wurden in anderen klinischen Studien zur Verträglichkeit von Weichspülern an insgesamt über 4000 Probanden - Testpersonen mit gesunder Haut sowie Atopiker, Neurodermitiker und Allergiker - gesehen (Rodriguez $C$ et al. Dermatosen in Beruf und Umwelt 42; 1994: 58-61. - Pièrard GE. Contact Dermatitis 30; 286-91. - Pièrard GE. Internat J Dermatol 33; 1994: 138-41).

Auch hier ergab sich, dass bei Personen mit geschädigter Haut wie auch in geringerem Umfang bei Personen mit gesunder Haut das Tragen von mit Weichspülern behandelter Wäsche zu einer Verbesserung des Hautzustandes führt.

\section{Mechanische Reibung auf der Haut vermindert}

Als Erklärung wird der „Wirkmechanismus" der Wäscheweichpfleger angeführt: Sie lagern u.a. Lipide an die Texilfaser an, die dort wie ein Gleitmittel auf der Haut wirken. Hierdurch wird das mechanische Reibungspotential der Wäsche deutlich verringert, was sich sowohl auf gesunde wie auch auf gereizte Haut positiv auswirkt (s. Abb.).

Insbesondere der Grad der durch wiederholtes Reiben von Textilien auf atopischer Haut hervorgerufenen Reizungen ist bei mit Weichspülern behandelten Stoffen geringer als bei Geweben ohne diese Zusatzbehandlung. Durch das geringere Irritationspotential erhöht sich somit gerade für atopische Patienten der Tragekomfort von weichgespülter Wäsche.

$b k$

\section{Bieber Th, et al.}

Publikation in Vorbereitung, Informationen nach einer Pressemitteilung der MedCom international, 1999. 